\title{
Lectin-based SERS Sandwich Immunoassay
}

\author{
Alexandra Olszewska ${ }^{1}$, Elizabeth Armstrong ${ }^{1,2}$, Maciej Olszewski ${ }^{1}$, Michael Batenjany ${ }^{1,3,4}$ and Agnes Ostafi ${ }^{1,2,3 *}$ \\ ${ }^{1}$ University of Utah, Nanolnstitute, Salt Lake City UT 84054, USA \\ 'University of Utah, Department of Chemical Engineering and Bioengineering, Salt Lake City UT 84054, USA \\ ${ }^{3}$ Nanoshell Company, LLC, North Salt Lake UT 84054, USA \\ ${ }^{4}$ University of Technology, Gdansk Poland 80-233, Poland
}

\begin{abstract}
Responding to need to develop sensitive and easy to use sensors for fungal diseases, we have shown using a portable Raman detector system that a SERS sandwich assay employing lectins can detect fungal cell wall polysaccharides at a level of $<200 \mathrm{pg} / \mathrm{ml}$ sensitivity. This is competitive with reported SERS sandwich immunoassays, and opens up a new approach to the development of recognition molecule-based sensors. Lectins, found throughout the microbial, plant and animal kingdoms target large polysaccharide structures, and could be used in multiplexed sensor designs to rapidly identify the nature of infection so that timely treatment can begin. SERS sandwich assays have the potential to detect analytes at concentrations as low as atto-molar. The combination of these two technologies has the potential for development of a wide range of useful sensing platforms for use in medicine and research.
\end{abstract}

\section{Keywords: SERS; Lectin; Immunoassay}

\section{Introduction}

Despite advances in pharmacological management, fungal diseases like Invasive Aspergillosis (IA) [1,2] are devastating, frequently fatal infections for immuno-compromised and immuno-suppressed patients (i.e. premature and malnourished infants, AIDS and cancer patients, seniors, and organ donation recipients) [3]. Diagnosis and initiation of antifungal therapy within the first 24 hours after innoculation can be a life or death determinant [4]. Today's enzyme-linked immunosorbent assays (ELISA) for Aspergillosis can detect fungal cell wall antigens at hundreds of $\mathrm{pg} / \mathrm{mL}$ or $\mathrm{ng} / \mathrm{mL}$ levels [5], but take several hours to perform and are compromised by interferences and patient-topatient variability. This work focuses on establishing the fundamental underpinnings for a new, lectin-based paradigm for Surface-Enhanced Raman Scattering (SERS) sandwich assay detection of disease markers, and demonstrating the feasibility of this approach for the detection of the fungal infection Aspergillosis. The antibody-based SERS immunosandwich assay has been demonstrated to be capable of femtomolarlevel detection of target antigens in 2-6 hours [6-8], which depending of the molecular weight of the marker can be a detection level significantly less than $\mathrm{pg} / \mathrm{mL}$ Lectin microarrays have been developed for basic research in glycomics analyses [9-11]. This recognition mode, however, has yet to be employed in the SERS sandwich assay.

The current immunological version of the SERS sandwich assay for galactomannan polysaccharide can reach limits of multiplex detection comparable or better than the corresponding ELISA kit, but is limited by the availability of effective antibodies, their stability and selectivity, and susceptibility to non-specific protein binding. Our hypothesis is that by implementing lectins in place of, or alongside antibodies, we can take advantage of lectin's unique features (i.e. multivalency, cooperative structural change, and catch-and-slip binding) to realize the greater potential of the SERS sandwich assay for polysaccharide detection. Then we can significantly impact the clinical outcome of affected patients by providing an easy to use, highly sensitive test that could quickly yield results and even be performed near the bedside using a portable detection system for more accurate and responsive care. Long-term, the technology we will develop can be extended to the detection of other fungal diseases that are a growing threat to global human, animal and plant health impacting ecosystems, agriculture, housing, food and medical product safety [12].
The detected Raman scattering signal in the SERS sandwich immunoassay [13] arises from the interaction of light with a specially selected Raman reporter molecule, such as dithiobis(succinimidyl propionate) attached to a gold nanoparticle (GNP) $\sim 60 \mathrm{~nm}$ in diameter. When this complex is localized within a few nanometers of an atomically smooth gold surface, coherence of surface plasmon resonances of the particle and the gold surface greatly amplifies (up to $10^{15}$ times) the Raman scattering signal [14]. The size of the nanoparticle is selected to maximize resonance coherence and the Raman reporter is selected to ensure that the signal is well-shifted from the naturally scattering, luminescent background of the specimen, usually of biological origin. In the SERS immuno-sandwich assay, both the gold surface and the GNP are decorated with antibodies and target antigens are trapped between the two. The length of crosslinkers for the recognition molecules is optimized to increase the signal strength [15]. The Raman signal is highly resolved (10-100 times narrower spectral linewidths than fluorescence emissions) and detectable under ideal conditions down to femtomolar antigen concentration in picoliters of sample [16], competing favorably with PCR based [17] methods. Examples of the SERS sandwich immunoassay have detected a variety of protein biomarkers in a broad range of diseases including tuberculosis [18], Dengue flu [19], fungal infections [20] and cancer [21].

While SERS assays have proven to be highly effective, ongoing challenges include availability of effective antibodies and limited understanding of molecular-events at the non-ideal assay interface and what conditions lead to interferences and non-specific binding. Antibody availability for fungal infections is a particular challenge because one of the primary biological functions of fungal cells is to circumvent $[22,23]$ or inhibit [24] the mammalian immune system

*Corresponding author: Agnes Ostafin, University of Utah, Nanolnstitute, Salt Lake City UT 84054, USA, Tel: 801-698-3955; E-mail: a.ostafin@utah.edu

Received October 15, 2016; Accepted October 25, 2016; Published October 31 2016

Citation: Olszewska A, Armstrong E, Olszewski M, Batenjany M, Ostafin A (2016) Lectin-based SERS Sandwich Immunoassay. J Nanomed Nanotechnol 7: 405. doi: 10.4172/2157-7439.1000405

Copyright: (C 2016 Olszewska A, et al. This is an open-access article distributed under the terms of the Creative Commons Attribution License, which permits unrestricted use, distribution, and reproduction in any medium, provided the original author and source are credited. 
from producing antibodies against them. Because one of the major antigenic markers of fungal infection are cell wall polysaccharides $[25,26]$ not present in mammalian cells, it is possible to use lectins instead of antibodies as a recognition element. Lectins are soluble proteins, non-enzymatic in function, that bind to soluble carbohydrates or to a carbohydrate component of a glycoprotein or glycolipid. There are more than 1000 highly effective and target-specific lectins $[27,28]$ throughout the animal [29,30], plant [31-33] marine [34] and fungal $[35,36]$ worlds where they play a protective role, and are involved in regulation of physiological functions and the recognition between cells or cells and various carbohydrate-containing molecules [37-40] They are used successfully in diagnostic and therapeutic applications to agglutinate specific cells and precipitate glycoconjugates. Jacalin, a plant lectin, is found to completely block human immunodeficiency virus type 1 in vitro infection of lymphoid cells, presumably by inducing the proliferation of $\mathrm{CD} 4^{+} \mathrm{T}$ lymphocytes [41].

Lectins (Figure 1) are generally smaller in size than antibodies (i.e. $<\sim 100 \mathrm{kDa}$ ). While antibodies have two single-point and independent antigen docking sites, lectins can have 4-12 binding sites on multiple subunits of the molecule and bind to target polysaccharides in multiple places with cooperativity [42]. In solution, this multivalent, complex binding has been described with the following model:

$$
K_{N}^{\text {poly }}=\left(K^{\text {Mono }}\right)^{\alpha N}
$$

Where $\alpha$ is the cooperativity factor and $\mathrm{N}$ is the number of binding interactions. Both the negative [43] and positive [44] cooperativity is found in lectin-carbohydrate systems, and is related to the lectin structural class and whether the event occurs in solution or on a cell surface. For some lectins $N$ can be as large as 10 and significantly larger than 1. So, while lectins bind individual monosaccharides weakly (dissociation constant $\mathrm{K}_{\mathrm{d}} \sim 10 \mathrm{uM}$ ), their affinity for larger, complex carbohydrate ligands with multiple connect points acting cooperatively can be significantly greater $[45,46]$. Many lectins also exhibit shearforce dependent catch-and-slip bond formation in which binding is improved when the molecules are exposed to fluid shear $[47,48]$. The phenomenon is best seen in the example of the binding of sialyl Lewis ${ }^{x}$
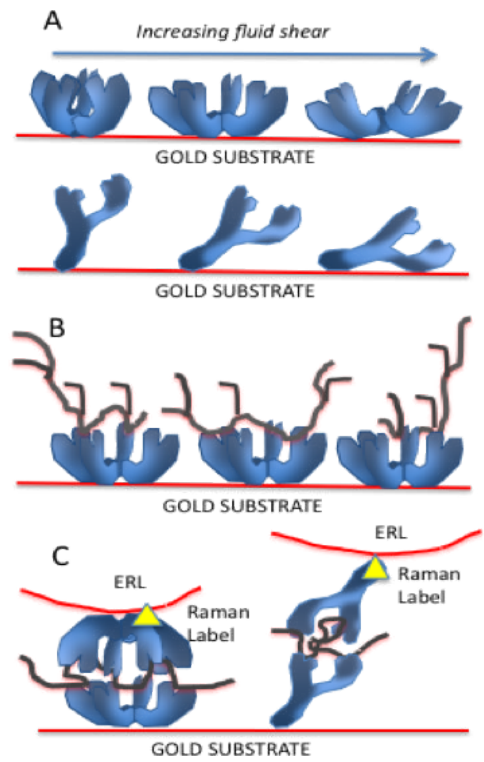

Figure 1: Unique features of lectin-polysaccharide recognition and sandwich assay. A) Effect of fluid shear on lectin and antibody configuration. B) Catchand-slip mechanism ( $L$ to R). C) Lectin vs. antibody SERS sandwich assay. (a tetrasaccharide attached to PSGL-1 on the surface of leukocytes) to selectin $\left(\mathrm{k}_{\text {on }} \sim 27000 \mathrm{M}^{-1} \mathrm{~s}^{-1}\right)$. The hinge domain of selectin opens and closes every few nanoseconds in solution, but under low shear stresses the open position is maintained allowing the catch-and-slip phenomenon to take place with sialyl Lewis molecules at different points in the chain [47]. This mechanism is responsible for the familiar rolling and slow down of leukocytes on the surface of blood vessels [48]. A major advantage of this feature is that a lectin-based SERS sandwich is likely to recognize and bind to targets better under constant fluid shear that occurs periodically during the sensor operation. Under similar conditions, fluid shear may degrade the performance of the immuno-based sandwich assay.

\section{Materials and Methods}

\section{Materials}

Gold surface preparation: A solid, acid-washed glass microscope slide, was sputter coated through a machined aluminum template with chromium to a thickness of $50 \mathrm{~nm}$, and then with gold to a thickness of $200 \mathrm{~nm}$ in order to obtain a microscopically smooth surface. The template allows the creation of a $3 \times 8$ array of gold dots, approximately 1 $\mathrm{mm}$ in diameter uniformly distributed across the slide (Figure 2). Prior to attachment of recognition molecules, the templated gold surface was peroxide-washed to remove any residual materials from the sputtering and dried in air in a dust free chamber. The templated gold microscope slide was them immersed in an ultrapure water solution containing 10 $\mathrm{mM}$ mercaptohexadecanoic acid (MHA) for 30 minutes, and then air dried in a dust free chamber.

OM-1 was attached to this surface via amide bonds. Om-1 is a human derived lectin that recognizes the galactofuranose moiety of galactomannan. The dry, MHA-coated support was briefly immersed in solution of EDC (1-Ethyl-3-(3-dimethylaminopropyl)-carbodiimide)/ S-NHS ( $N$-hydroxysulfosuccinimide) according to the manufacturer's instructions. This formed amide bonds between the MHA and EDC molecules, and presented a reactive succinimidyl derivative on the support surface for reaction with an exposed amine group on OM-1. The slide was then incubated in phosphate buffer solutions of OM-1, followed by rinse with ultrapure water. Unreacted sites were blocked with $150 \mathrm{ul}$ TBST per well $\mathrm{x} 2 \mathrm{hr}$.

Gold nanoparticle preparation: Gold nanoparticles $100 \mathrm{~nm}$ in diameter were mixed with an ultrapure water solution containing 1 $\mathrm{mM}$ disuccinimidyl nitrobenzoate (DSNB) titrated with $\mathrm{NaOH}$ to $\mathrm{pH}$ 8. DSNB contains two Raman active nitroso groups ( $\boldsymbol{v}_{\mathrm{s}} 1336 \mathrm{~cm}^{-1}$ shift) two succinimidyl group for reaction with lectin, and a disulfide bond. The disulfide bond is reduced at the gold nanoparticle surface and the
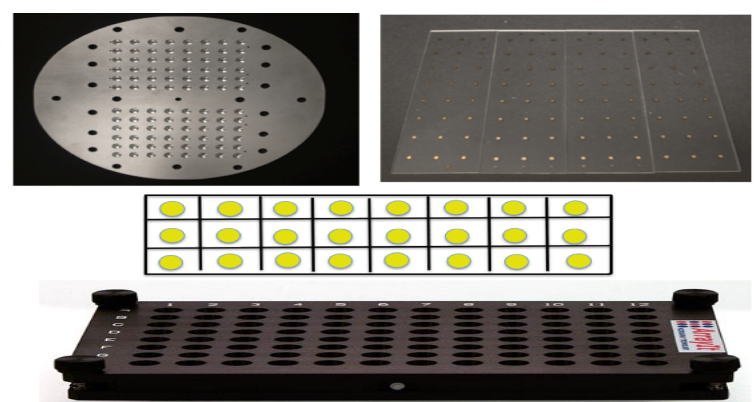

Figure 2: Top right: Aluminum sputtering mask; Top left: Microscope slides after deposition of gold. Middle: schematic of arrangement of gold island on the microscope slide. Bottom: assembled assay device. 
two halves of DSNB each are available to bind to the gold nanoparticle via an $\mathrm{Au}-\mathrm{S}$ ligand bond which is extremely stable. Concavalin A was added to the mixture and allowed to react for 15 minutes with the available succinimidyl groups. Unbound DSNB, DSNB fragments, and Concavalin A were removed by centrifugal pelleting of the functionalized gold nanoparticles, decanting of the supernatant, and resuspension of the nanoparticles in ultrapure water. The decanted fraction was reserved for spectroscopic analysis to obtain information about the binding process.

Assay assembly: The Array-It 96-well plate microwell plate assembly was adopted for the assay (Figure 2 bottom image). This device consists of two plastic parts into which up to 4 gold-templated, OM-1 functionalized microscope slides can be inserted and the two halves pressed together with a silicone gasket in between. The upper plate created the well into which assay reagents and specimen were applied. Greer's antigen (which contains $\sim 10 \%$ by weight of the A. fumigatus galactomannan) was added in triplicate from 0-64 ng per gold spot. To complete the detection sandwich, Concavalin A functionalized 100 $\mathrm{nm}$ gold particles were applied and the excess particles rinsed using ultrapure water

\section{Material characterization}

UV-Vis absorption measurements were carried out using a Cary 50 (Agilent) in $1 \mathrm{~cm}$ pathlength microcuvettes at room temperature. Dynamic light scattering measurements of gold nanoparticle was performed using a Zetasizer ZS (Malvern) in semi micro cuvettes at $25^{\circ} \mathrm{C}$. SERS signals generated by the nitroso group of DSNB $\left(\mathrm{V}_{\mathrm{s}}\right.$ at $1336 \mathrm{~cm}^{-1}$ Raman shift) were measured either using the MiniRAM III (BWTEK, Newark, DE), which utilizes a $785 \mathrm{~nm}$ laser and is capable of $10 \mathrm{~cm}^{-1}$ resolution of Raman shifts or MiniRam (BW-Tek) instrument was used with a $785 \mathrm{~nm}$ laser set to $40 \%$ power level $(\sim 150 \mathrm{~mW})$ and a $10 \mathrm{msec}$ integration time. Data was collected at multiple spots in each well, and averaged. The dark current was subtracted from all spectra.

\section{Results and Discussion}

Surface-enhanced Raman scattering (SERS) is a powerful technique for analyzing biological samples as it can rapidly and nondestructively provide chemical and, in some cases, structural information about molecules in aqueous environments. In the Raman scattering process, both visible and near-infrared (NIR) wavelengths of light can be used to induce polarization of Raman-active molecules, leading to inelastic light scattering that yields specific molecular vibrational information. The development of surface enhancement has enabled Raman scattering to be an effective tool for qualitative as well as quantitative measurements in immunoassays with high sensitivity and specificity.

In the SERS sandwich all-lectin assay, a lectin specific for a target polysaccharide molecule is immobilized on a surface via succinimidyl ester chemistry to a gold-bound thiolate adlayer formed from dithiobis(succinimidyl propionate) (DSP). When target is present, the immobilized layer of lectin specifically extracts available target from the surrounding fluid. After rinsing, the surface is then incubated with Extrinsic Raman Labels (ERLs). These are nanoparticles of gold decorated with 5,5'- dithiobis(succinimidyl-2-nitrobenzoate) (DSNB). The nitro group of the bound DSNB provides an intense SERS signal, while the succinimide group acts as a coupling agent for tethering another lectin to the nanoparticle. The quantity of target sandwiched between the two lectins in a given area is estimated by the spectral intensity of the symmetric nitro stretch $\left(v_{s}\left(\mathrm{NO}_{2}\right)\right)$. Ideally, there would be one sandwich per ERL on the gold surface, but this is difficult to control. Thus there is some inherent quantitative uncertainty due to variable connection points per particle.

During the assembly it is possible to monitor each step to ensure optimal conditions. For example, effectiveness of DSP and DSNB binding in a given area of the platform can be estimated spectroscopically by monitoring the fraction of material left unbound. The uniformity of lectin binding to the surface can be measured using SEM. For control samples, the amount of bound target can be confirmed via the residual amount of target remaining in the fluid, but, for unknown samples it is necessary to rely on an experimentally-derived estimate of SERS signal intensity per ERL nanoparticle. This method has some drawback in that for high target titers the attachment density of ERLs is also high and so nanoparticle-nanoparticle interactions can alter the effective signal intensity per nanoparticle signal. Thus this technique worked best at low target concentrations, where the distance between attached ERL nanoparticles is large.

The all-lectin SERS sandwich assay utilizing Om-1 on the flat gold substrate and Concavalin A on the gold nanoparticle show sensitivities of less than $100 \mathrm{pg}$ of A. fumigatus galactomannan. Typical signals are shown in Figure 3. The galactomannan used is in the form of an extract mixture of cell wall components from the organism and comprises $<10 \%$ of total material. We will refer to this mixture subsequently as Greer's antigen. Typical SERS signals recorded as a function of total amount of Greer's antigen (in ng) are shown in Figure 3. Figure 4 shows

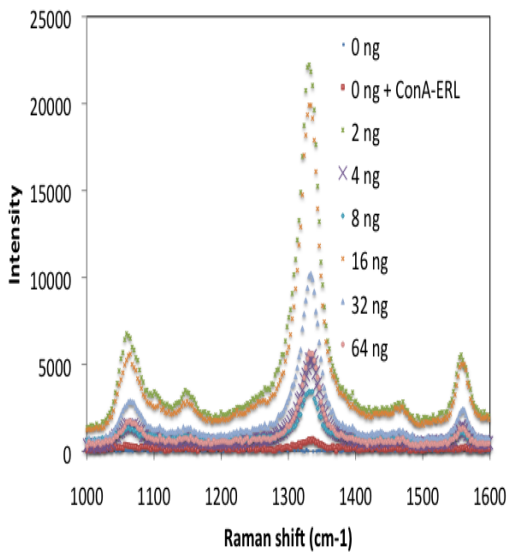

Figure 3: Typical Raman shift spectra observed from the lectin-lectin sandwich assay as a function of Greer's antigen concentration. Greer's antigen contains less than $10 \%$ galactomannan, the remaining being other cell wall glycoproteins.

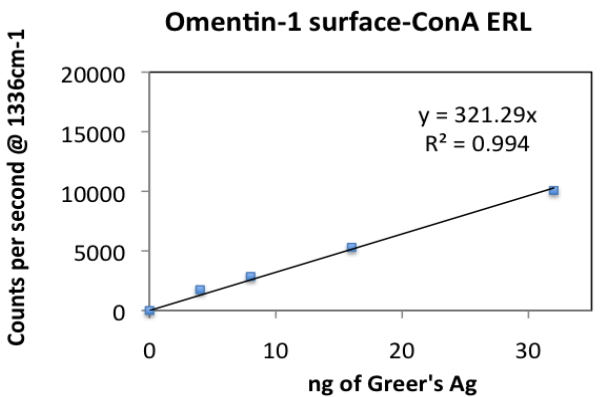

Figure 4: Background-subtracted SERS signal intensity of an all lectin SERS sandwich assay in which Om-1 is used in place of antibody clone WF-AF-1. The percentage of galactomannan present is about $10 \%$ by weight suggesting a current sensitivity of detection of about 100 picograms. 
a galactomannan dose-response curve with excellent linearity. The data were corrected for instrument baseline signal as well as background scattering. The lowest detectable amount of galactomannan is represented at the origin of the graph, and is less than 100 pmol. The signal intensity represented on the $\mathrm{Y}$ axis is both instrument and assay dependent. The important point to note is that the BWTek device is intended for use as a portable field unit, thus this level of sensitivity is excellent.

Shown in Figure 5 are results obtained using a SERS sandwich immunoassay using commercially available WF-AF-1 antibody, as well as those from the Platellia EIA kit used clinically. The overall intensity axis of this graph is smaller than that in Figure 4, due to use of a different instrument and slightly different gold nanoparticles in the assembly. Both these systems provide similar quality dose-response curves with a limiting sensitivity of about 100 picograms galactomannan content. This shows that the all-lectin SERS sandwich assay and the conventional antibody-based SERS sandwich immunoassay can be used interchangeably with loss of sensitivity.

Two other galactomannan binding lectins Lens Culinaris Agglutinin (LCA) and Hippeastrum Hybrid Lectin (HHL) were compared to Concavalin A in the all-lectin SERS sandwich assay (Figure 6). All three lectins respond more or less linearly to the presence of analyte, but Concavalin A generates the largest signals as a function of increasing galactomannan concentration under the same conditions. Both exhibit a gradual increase in signal with continued linearity.

As seen in Figure 5 the actual working range for Concavalin A ERLs is relatively narrow, with SERS signals plateauing above about $32 \mathrm{ng} / \mathrm{ml}$ of Greer antigen mixture. This is mainly due to the signal collecting limitations of the hand-held Raman device. In the field,

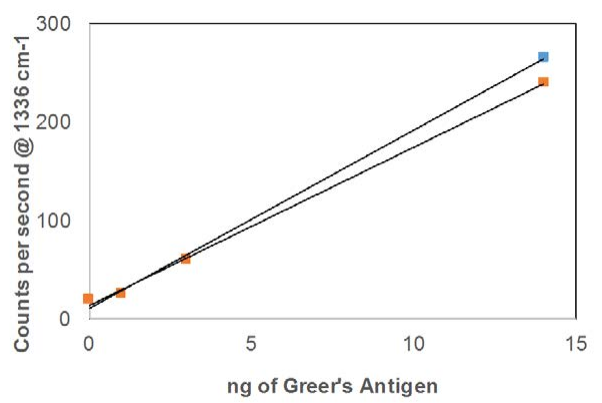

Figure 5: SERS immunoassay using WF-AF-1 antibody (red squares) and Platelia $^{\mathrm{TM}}$ EIA (Black squares) for galactomannan in Greer's Ag. Courtesy M. Porter.

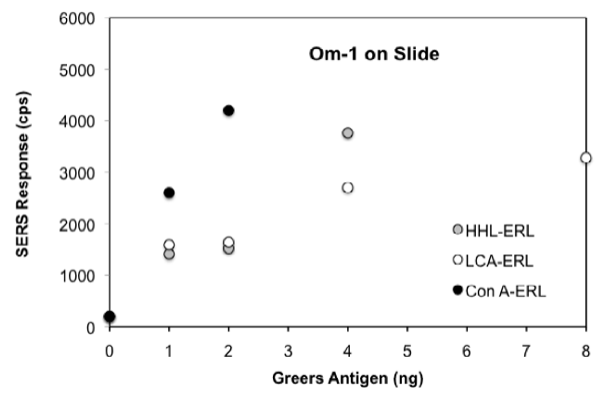

Figure 6: SERS signal intensity of Om-1/various lection ERL systems. where there may be a wide range of analyte concentrations possible, having a sensor that is limited to only the lowest concentration ranges can be cumbersome. If a sensor analyzes the same unknown sample simultaneously using two types of lectins, for example Concavalin A for the lower range and Lens Culinaris Agglutinin for a higher range, the array employing LCA, for instance will continue to report a reliable linear signal even when the Concavalin A section of the array is no longer reliable. The two measurements together provide a cross-check of each other as well in overlap regions. The data shown the linearity of response for Lens Culinaris Agglutinin and Hippeastrum Hybrid Lectin is poorer than for OM-1. This is due in part to the complex structure of those proteins which makes binding affinity and specificity slightly different than for OM-1. In a multiplexed platform, errors at low concentration are covered by OM-1. In general it is necessary to pick the best lectin pair to construct an optimal assay under the desired conditions.

\section{Acknowledgements}

Thanks to Dr. Marc Porter, University of Utah Nanolnstitute for use of Raman detection equipment and information reqarding SERS sandwich immunoassays.

\section{References}

1. Pagano L, Caira M, Candoni A, Offidani M, Fianchi L, et al. (2006) The epidemiology of fungal infections in patients with hematologic malignancies: the SEIFEM-2004 study. Haematologica 91: 1068-1075.

2. McNeil MM, Nash SL, Haijeh RA, Phelan MA, Conn LA, et al. (1998) Trends in mortality due to invasive mycotic diseases in the United States. International Conference on Emerging Infectious Diseases. Atlanta Georgia 641-647.

3. Bow EJ (2009) The role of second-generation triazole antifungal agents voriconazole and posaconazole in patients with hematologic malignancies. Curr Fungal Infect Rep 3: 32-39.

4. Hope WW, Petraitis V, Petraitiene R, Aghamolia T, Bacher J, et al. (2010) The Initial 96 Hours of Invasive Pulmonary Aspergillosis: Histopathology, Comparative Kinetics of Galactomannan and $(1 \rightarrow 3)-\beta-d-$-Glucan, and Consequences of Delayed Antifungal Therapy. Antimicrob Agents Chemother 54: $4879-4886$

5. http://www.bio-rad.com/webroot/web/pdf/cdg/literature/J115_Microbiology.pdf

6. Driskell JD, Kwarta KM, Lipert RJ, Porter MD (2005) Low-Level Detection of Vira Pathogens by a Surface-Enhanced Raman Scattering Based Immunoassay. Anal Chem 77: 6147-6154.

7. Jiang J, Bosnick K, Maillard M, Brus L (2003) Single molecule Raman spectroscopy at the junctions of large Ag nanocrystals. J Phys Chem B 107: 9964-9972.

8. Ca YC, Rongchao J, Mirkin CA (2002) Nanoparticles with Raman Spectroscopic Fingerprints for DNA and RNA Detection. Science 297: 1536

9. Campbell C, Gildersleeve J (2011) Tools for glycomics: Glycan and Lectin Microarrays. In Carbohydrate Recognition. Biological Problems, Methods and Applications. Wiley Hobokken, New Jersey.

10. Yonzon CR, Jeoung E, Zou S, Schatz GC, Mrksich M, et al. (2004) A comparative anaylsis of localized and propagating surface Plasmon resonance sensors: The binding on concavalin A to a monosaccharide functionalized selfassembled monolayer. J Amer Chem Soc 126: 12669-12676.

11. Vangala K, Yanney M, Hsiao CT, Wu WW, Shen RF, et al. (2010) Sensitive Carbohydrate Detection Using Surface Enhanced Raman Tagging. Anal Chem 82: 10164-10171.

12. Olsen L, Choffnes E, Relman D, Pray L (2011) Fungal diseases: An emerging threat to human, animal and plant health. Workshop Summary, Forum on Microbial threats; Board on Global Health. National Academies Press Washington DC, USA.

13. Ni J, Lipert RJ, Dawson GB, Porter MD (1999) Immunoassay Readout Method Using Extrinsic Raman Labels Adsorbed on Immunogold Colloids. Anal Chem 71: 4903-4908.

14. Moskovits M (20006) Surface-enhanced Raman spectroscopy: A brief perspective. Topics Appl Phys 103: 1-18. 
Citation: Olszewska A, Armstrong E, Olszewski M, Batenjany M, Ostafin A (2016) Lectin-based SERS Sandwich Immunoassay. J Nanomed Nanotechnol 7: 405. doi: 10.4172/2157-7439.1000405

Page 5 of 5

15. Grubisha DS, Lipert RJ, Park HY, Driskell J, Porter MD (2003) Femtomolar Detection of Prostate-Specific Antigen: An Immunoassay Based on SurfaceEnhanced Raman Scattering and Immunogold Labels. Anal Chem 75: 5936-5943.

16. White PL, Barton R, Guiver M, Linton CJ, Wilson S, et al. (2006) A consensus on fungal polymerase chain reaction diagnosis. J Molec Diagnos 8: 376-384.

17. Crawford AC, Granger J, Chatterjee D, Quinn FD, Hanson K, et al. (2013) Multiplexed detection of Mycobacterium tuberculosis antigens using a surfaceenhanced Raman scattering immunoassay platform. 245 Metting and Exposition, New Orleans, LA.

18. Chen Y, Pong Z, Siperko L, Porter M (2008) Low-level detection of Aspergillus antigen Galactomannan in serum using surface enhanced Raman Scattering (SERS). MS in preparation.

19. Granger JH, Granger MC, Firpo MA, Mulvihill SJ, Porter MD (2013) Toward development of a surface-enhanced Raman scattering (SERS)-based cancer diagnostic immunoassay panel. Analyst 132: 410-416.

20. Wheeler RT, Kombe D, Agarwala SD, Fink GR (2008) Dynamics and morphotype-specific candida Albicans b-glucan expsure during infection and drug treatments. Plos Pathogens 4: 1-12.

21. Fujikawa T, Sakaguchi A, Nishizawa Y, Kouzai Y, Minami E, et al. (2012) Surface alpha-1,3, glucans facilitates fungal stealth infection by interfereing with innate immunity in plants. Plos Pathogen 8:8.

22. Yauch LE, Lam JS, Levitz SM (2011) Direct inhibition of T-Cell responses by the Cryptococcus capsular polysaccharide glucuronoxylomannan. Plos Pathogen 1060-1068.

23. Rodriguez ML, Nimrichter L, Cordero RJB, Casadevall A (2011) Funga polysaccharides:biological activity beyond the usual structural properties. Frontiers in Microbiology 2: 1-4.

24. Latge JP (2007) The cell wall: a carbohydrate armour for the fungal cell. Molec Microbiol 66: 279-290.

25. http://lectin3d.cermav.cnrs.fr/search.php

26. http://www.google.co.in/url?sa $=t \& r c t=j \& q=\&$ esrc $=s \&$ source $=$ web \&cd $=1 \& v e d=0$ ahUKEwjg1NCuvIfQAhWHQY8KHaFNCfAQFggbMAA\&url=http $\% 3 A \% 2 F \% 2 F$ www.ajol.info\%2Findex.php\%2Fajb\%2Farticle\%2Fdownload\%2F120425\%2F 109901\&usg=AFQjCNGA5IXVy5mpjO3xKB1DUp3kOmflqw\&bvm=bv.137132 246 , d.c2I\&cad=rja

27. Kilpatrick DC (2002) Animal Lectins: a historical introduction and overview. Bichim Biophys Acta 1572: 187-197

28. Gabius HJ (2009) Animal and Human Lectins in the Sugar Code Fundamentals of Glycosciences. Weinhein, Wiley VCH Chap 19: 317-328.

29. Lorenc-Kubis I (1998) Lectins of higher plants and their carbohydrate-binding specificity. Wiadomoscis Botaniczne 42: 21-33.

30. Ruediger H, Gabius HJ (2009) Plant Lectins in The Sugar Code Fundamentals of Glycosciences. Weinhein, Wiley VCH Chap 19: 301-315

31. De Hoff, Brill PL, Hirsch LM (2009) Plant Lectins: the ties that bind in root synthesis and plant genomics, Mol Genet Genomics 282: 1-15.
32. Ogawa T, Watanabe M, Naganuma T, Muramoto K (2011) Diversified Carbohydrate-Binding Lectins from Marine Resources. J Amino Acids.

33. Khan F, Khan MI (2011) Fungal lectins: Current molecular and biochemical perspectives. Inter J Biological Chem 5: 1-20.

34. Shalhanina KL, Kalinin NL, Lakhtin VM (1994) Microbial lectins for the investigation of glycoconjugates. Lectin-Microorganism Interactions 1: 299-326.

35. Mansour MK, Latz E, Levitz SM (2006) Cryptococcus neoformans Glycoantigens are captures by multiple lectin receptor and presented by dendritic cells. J Immunol 176: 3053-3061.

36. Lakhtin V, Lakhtin M, Alyoshkin V (2011) Lectins of living organisms: the overview. Anaerobe, 17: 452-455

37. Kiran KK, Lalith PCK, Sumanthi J, Reddy S, Chandra SP, et al. (2012) Biological role of lectins. J Orofacial Sci 4:1

38. Barondes SH (1981) Lectins: Their multiple endogenous cellular functions. Annual Review of Biochemistry 50: 207- 231.

39. Favero J, Corbeau P, Nicolas M, Benkirane M, Trave G, et al. (1993) Inhibition of human immunodeficiency virus infection by the lectin jacalin and by a derived peptide showing a sequence similarity with gp120. Euro J Immunology 23: 179-85.

40. Brown A (2009) Analysis of cooperativity by isothermal titration calorimetry. Int J Mol Sci 10: 3457-3477.

41. Rao DH, Gowda LR (2012) The d-galactose specific lectin of field bean (Dolichos lablab) seed binds sugars with extreme negative cooperativity and half-of-the-sites binding. Archives Biochem Biophys.

42. Ermakova E, Miller MC, Nesmelova IV, Lopez-Merino L, Berbis, et al. (2013) Lactose Binding to Human Galectin-7 (p53-induced gene 1) Induces LongRange Effects through the Protein Resulting in Increased Dimer Stability and Evidence for Positive Cooperativity. Glycobiology 23: 508-523.

43. Rini JM (1995) Lectin Structure Ann Rev. Biophys Biolmolec Struct 24: 551-577.

44. MacKenziec CR, Hirama T, Deng SJ, Bundle DR, Narang SA, et al. (1996) Analysis by Surface Plasmon resonance of the influence of valence on the ligand binding affinity and kinetics of an anti-carbohydrate antibody. J Biological Chem 271: 1527-1533.

45. Beauharnois ME, Lindquist KC, Marather $D$, Vanderslice $P$, Matta $K L$, et al (2005) Affinity and kinetics of sialyly Lewis X and Core-2 based Oligosaccharide binding to L- and P-selectin. Biochem 44: 9507-9519.

46. Nimrichter L, Burdick MM, Aoki K, Laroy W, Fierro MA, et al. (2008) E-selectin receptors on human leukocytes. Blood 112: 3744-52.

47. Thomas W (2006) For Catch Bonds, it all hinges on the interdomain region. $J$ Cell Biol 174: 911-913.

48. Cotran C, Kumar C (1998) Robbins Pathologic Basis of Disease. Philadelphia W.B Saunders Company. ISBN 0-7216-7335-X. 